CONTEXT DIVERSITY PREDICTS WEAPON BIAS DECLINE

\author{
Implicit Black-Weapon Associations Weakened Over Time in Increasingly Multiethnic \\ Metropolitan Areas \\ Angela Somo \\ Melody Sadler \\ Thierry Devos \\ San Diego State University
}

Paper accepted for publication in Analyses of Social Issues and Public Policy on 10/21/2020

\begin{abstract}
Author Note
Study materials and full datasets used for the present research (https://osf.io/f4htw/) as well as analysis scripts and data files necessary to reproduce the findings (https://osf.io/vmy7b/) are publicly available. This research was part of Angela Somo's thesis, completed at San Diego State University. The plan of analysis was not preregistered.

Correspondence concerning this article should be addressed to Melody Sadler, Department of Psychology, San Diego State University, 5500 Campanile Drive, San Diego, CA 92182-4611. Email: msadler@sdsu.edu.
\end{abstract}

Word count text: 8,251 words

Word count abstract: 163 words 
CONTEXT DIVERSITY PREDICTS WEAPON BIAS DECLINE

\author{
Abstract \\ A repeated cross-sectional design was used to examine whether temporal changes in implicit \\ Black-weapon associations were dependent on the changing ethnic diversity of metropolitan \\ areas over the course of a decade (2009-2018). Data on implicit Black-weapon associations were \\ obtained from Project Implicit. Three indicators of ethnic diversity were calculated using \\ American Community Survey data. Minority representation referred to the proportion of African \\ American residents. Variety was operationalized as the degree to which six ethnic groups each \\ accounted for an equal proportion of the population. Integration assessed the degree to which \\ ethnic groups were evenly distributed across census tracts making up the metropolitan area. \\ Multilevel model analyses $(N=345,647$ participants, nested within 185 metropolitan areas) \\ revealed that implicit Black-weapon associations weakened over time, and to a larger degree in \\ metropolitan areas characterized by steeper increases in variety. This longitudinal relationship is \\ consistent with the notion that, as metropolitan areas become more multiethnic, implicit \\ associations between crime, danger, or violence and Black Americans decline. \\ Keywords: automatic, implicit, stereotypes, weapon, diversity, time
}


CONTEXT DIVERSITY PREDICTS WEAPON BIAS DECLINE

\section{Implicit Black-Weapon Associations Weakened Over Time in Increasingly Multiethnic Metropolitan Areas}

Prompted by police shootings of unarmed Black Americans, along with public outcries and mass media attention covering these deaths, researchers have examined implicit racial biases affecting assumptions about criminal activity (for reviews, see Correll et al., 2014; Payne, 2006; Sommers \& Marotta, 2014; Spencer et al., 2016). There is compelling evidence that concepts such as weapon, crime, danger, or violence are more strongly associated with Black Americans than with White Americans (Correll et al., 2002; Greenwald, Oakes, et al., 2003; Mekawi \& Bresin, 2015; Payne, 2001; Plant \& Peruche, 2005). Recent research showed that implicit Blackweapon associations are weaker in more ethnically diverse metropolitan areas (Sadler \& Devos, 2020), but the extent to which they fluctuate across time has not been examined yet. Is this implicit stereotype declining or increasing? Does it vary as a function of how ethnically diverse the context is? Building on recent streams of research, our goal was to test whether changes in the ethnic diversity of metropolitan areas accounted for variations in implicit Black-weapon associations.

Implicit Black-weapon associations have been documented using a variety of techniques including the First-Person Shooter Task (FPST, Correll et al., 2002), the Weapons Identification Task (WIT, Payne, 2001), and the Implicit Association Test (IAT, Nosek et al., 2007). Setting asides nuances among theoretical perspectives and paradigms, the link between weapons and Black (vs. White) Americans manifests itself in automatic assumptions operating when cognitive control is hindered (Payne et al., 2002), in different thresholds to decide whether an individual is armed or not (Correll et al., 2002), and in how ambiguous stimuli are perceived (Eberhardt et al., 2004) to name a few. Researchers have made strides towards understanding factors that influence 
CONTEXT DIVERSITY PREDICTS WEAPON BIAS DECLINE

the degree, accessibility, or impact of Black-weapon associations. A lot of attention has been dedicated to immediate contextual cues (e.g., background of environment, target prototypicality, individual vs. group situation) (Cooley et al., 2019; Correll et al., 2011; Ma \& Correll, 2011). In addition, perceivers' characteristics also account for the magnitude of Black-weapon associations. For example, the effect is weaker among Black participants than among members of other ethnic groups (Nosek et al., 2007).

More recently, researchers have started to examine the role that the larger, societal context may play in implicit biases (Murphy et al., 2018; Payne et al., 2017). In this vein, several studies have documented relationships between characteristics of contexts and implicit biases (Payne et al., 2019). Notably, Hehman et al. (2018) found that geographical areas where implicit racial biases of White residents (Black-weapon associations and anti-Black attitudes) were stronger were also areas where the disproportionate use of lethal force by police officers was more pronounced. Similarly, Johnson and Chopik (2019) observed that in U.S. states where Black (compared to White) people were more often killed by firearms, implicit (and explicit) associations between weapons and Black people were stronger.

Most directly relevant to the present research, the ethnic diversity of the context accounts for the strength of implicit Black-weapon associations. Building on conceptual and empirical developments in the social science literature (Koopmans \& Schaeffer, 2015; Reardon \& Firebaugh, 2002), Sadler and Devos (2020) examined the role of three dimensions of context diversity assessed at the level of metropolitan areas (see also Devos \& Sadler, 2019). Minority representation referred to the proportion of African American residents in a given metropolitan area. Variety mapped the extent to which the metropolitan area was multiethnic in the sense that various ethnic groups (e.g., African, Asian, European, and Latinx Americans) accounted for a 
CONTEXT DIVERSITY PREDICTS WEAPON BIAS DECLINE

similar share of its population. Integration captured the extent to which these ethnic groups were distributed evenly across sub-areas making-up the broader context. Specifically, integration would characterize contexts where variety is similar at the broader (metropolitan area) level and within each sub-areas (census tracts), whereas segregation would refer to contexts where some ethnic groups are overrepresented or underrepresented in some sub-areas (census tracts), implying unevenness in the spatial distribution of ethnic groups compared to the ethnic make-up of the metropolitan area as a whole. The results showed that the tendency to associate weapons with Black Americans compared to White Americans was weaker when ethnic groups were more proportionally represented in metropolitan areas (higher variety) and the distribution of the ethnic groups was isomorphic at census tract and metropolitan area levels (higher integration) (Sadler \& Devos, 2020). Minority representation only qualified the effect of integration: integration was associated with weaker Black-weapon associations when the representation of African Americans was low, but not when it was high.

These cross-sectional relationships between context diversity and implicit Blackweapon associations are consistent with the notion that implicit biases reflect the knowledge accessible in a given context. Characteristics of the context in which individuals are immersed shape their knowledge and experiences. Implicit associations about race-related issues are likely to stem from exposure to media coverage about crime reports, police brutality, or collective protests, but they may also be anchored in everyday interactions with others, exposure to ideas, practices, symbols, or products that bear the mark of historical relations between ethnic groups. Some of these factors may operate relatively uniformly, thereby making this knowledge highly accessible across contexts (e.g., disproportionate representations of Blacks as criminals and Whites as victims of crimes in news reports). At the same time, characteristics of more local 
CONTEXT DIVERSITY PREDICTS WEAPON BIAS DECLINE

contexts also determine the accessibility of specific knowledge and thus may contribute to variations in implicit associations across contexts or situations. Furthermore, the knowledge accessible may fluctuate across time as events unfold, become more salient, or are more likely to be discussed. For example, media coverage of specific events or societal topics may differ across contexts and over time. Directly relevant to Black-weapon associations would be highly publicized cases of police brutality and the collective actions and protests that unfolded. Being exposed to and processing this information is likely to strengthen or undermine implicit associations depending on the context in which individuals are immersed.

In the present research, we focused specifically on the role that context diversity may play in implicit Black-weapon associations. In light of earlier findings suggesting that variety and integration (rather than minority representation) account for this specific implicit bias (Sadler \& Devos, 2020), we posited that these two forms of context diversity attenuate the salience of the Black-White divide. The presence of multiple ethnic groups in a context highlights finer distinctions (high variety). As a result, the context is less likely to be appraised according to a dichotomous frame. Over the course of experiences and interactions, multiple categorical distinctions or identities are accessible. A relatively complex categorical system may help undermine stereotypical associations compared to when Black and White Americans are contrasted to one another. The accessibility of the Black-White divide may also be reduced when ethnic groups are evenly distributed across sub-areas making up the broader context (high integration). Integration may dilute the visibility of an ethnic group in particular when this group accounts for a relatively small percentage of the population.

Up until now, little research has examined how changes in socio-structural factors might account for implicit biases. However, researchers have tested whether average levels of implicit 
CONTEXT DIVERSITY PREDICTS WEAPON BIAS DECLINE

biases change over time. Findings on this issue are mixed; they seem to depend on the attitude or stereotype studied as well as on the data analysis strategy. Regressing racial attitudes on data collection date (using participant level data and controlling for participant demographics), it was found that implicit (and explicit) racial attitudes changed minimally during Barack Obama's presidential campaign and early presidency (Schmidt \& Nosek, 2010). No substantial changes were observed later into Obama's tenure as president (Schmidt \& Axt, 2016). Using a regression discontinuity analysis, Sawyer and Gampa (2018) found that Whites' implicit attitudes were less pro-White during the Black Lives Matter (BLM) movement compared to before the BLM movement. A study examining attitudes based on sexual orientation showed that negative implicit attitudes towards sexual minorities declined by 13.4\% from 2007 to 2013 (Westgate et al., 2015). Another study examining the impact of the Ebola crisis on implicit attitudes based on sexual orientation yielded inconclusive findings (Inbar et al., 2016).

A limitation to the aforementioned studies is the choice of statistical analysis. Applying regression to analyze change in implicit bias over time is likely to violate assumptions of linearity and independence (Charlesworth \& Banaji, 2019; Jebb et al., 2015). Specifically, implicit biases measured on two consecutive days are more likely to be similar compared to implicit biases measured on any two random days in a year. A pioneer study by Charlesworth and Banaji (2019) addressed the issue of autocorrelation by utilizing a time series approach to investigate change in implicit bias over time. The comparative time series analysis of six attitude tasks available on Project Implicit showed that implicit sexual orientation, race, and skin-tone attitudes shifted over time and were now approaching neutrality (i.e., no preference for one social group compared to the other), whereas implicit age, weight, and disability attitudes remained relatively stable over time. The rate at which implicit biases based on sexual 
CONTEXT DIVERSITY PREDICTS WEAPON BIAS DECLINE

orientation, race, and skin-tone declined varied, with implicit sexual orientation attitudes approaching neutrality the quickest and skin-tone attitudes the slowest. These descriptive findings highlight heterogeneity in patterns of change. The authors suggested that decline in implicit biases might be more likely on topics that received greater public attention and were extensively discussed at a societal level.

There is little doubt that the role of race in policing has received increased societal attention over the last two decades, but changes in implicit Black-weapon associations have not been examined yet. Documenting societal shifts in implicit biases is important, but temporal fluctuations may not be uniform across contexts. Depending on the changing nature of contextual characteristics, different patterns of implicit associations are likely to unfold. Cross-sectional data do not shed light on issues of change and cannot be used to infer longitudinal relationships between explanatory and dependent variables (Ruspini, 1999). Nonetheless, the notion that implicit associations reflect the knowledge accessible in a given context at a particular point in time may account for both cross-sectional and longitudinal variations. Extrapolating from previous findings (Sadler \& Devos, 2020), one would expect that as metropolitan areas increased in variety and integration over time, implicit Black-weapon associations correspondingly decreased over time.

It is important to point out that stability and fluctuation over time are not mutually exclusive. The stability of context-level implicit biases may result from the inertia of sociostructural factors or from the long-lasting influences of socio-historical circumstances. This perspective is supported by Payne et al. (2019) who found that the proportion of slaves in a county in 1860 predicted current levels of implicit racial attitudes. At the same time, contexts are not inert and to the extent that they change over time, variations in implicit biases might be 
CONTEXT DIVERSITY PREDICTS WEAPON BIAS DECLINE

detectable. For example, a recent study showed that patterns of change in context diversity over three decades (1980 to 2010) predicted current levels of implicit associations between the American identity and European vs. Asian Americans (Devos et al., 2019). Steeper increases in the proportion of Asian Americans at the level of metropolitan areas over the course of 30 years accounted for weaker implicit American $=$ White associations at present time . In addition, implicit American = White associations were stronger when integration increased recently rather than more distally within this period. Although changes in context diversity were related to current levels of implicit bias rather than to changes in levels of implicit bias, these findings point to the importance of adding a temporal dimension to investigations of the socio-structural foundations of implicit biases.

\section{Research Overview}

The aims of the present research were to test whether implicit Black-weapon associations changed over a 10-year period and whether changes in context diversity could at least partially account for these temporal fluctuations. Temporal fluctuations in Black-weapon associations have not been examined so far. In light of prior research (Charlesworth \& Banaji, 2019) and increasing societal discussions around the role of race in the criminal justice system, we hypothesized that implicit Black-weapon associations weakened over the last 10 years (Hypothesis 1). Next, we predicted that this decline would not be uniform across metropolitan areas. Specifically, we hypothesized that implicit Black-weapon associations weakened to a greater extent in metropolitan areas that became more multiethnic (Hypothesis 2) and more integrated (Hypothesis 3) over time. In other words, in addition to cross-sectional effects associated with variety and integration, temporal fluctuations of these two forms of context diversity should account for temporal fluctuations in implicit Black-weapon associations. To our 
CONTEXT DIVERSITY PREDICTS WEAPON BIAS DECLINE

knowledge, no prior research has shown that temporal fluctuations in implicit biases vary across contexts. Thus, an important contribution of the present research is to test whether implicit biases declined at various paces in different contexts and to determine whether these variations can at least partially be accounted for by two specific forms of context diversity (variety and integration). As metropolitan areas became more ethnically diverse in terms of variety or integration, a corresponding decline of implicit stereotypes should unfold. Although the present study was not designed to provide direct evidence for specific mechanisms underlying these relations, they would be consistent with principles put forth to account for cross-sectional relations. As variety increases over time in a metropolitan area, distinctions among multiple ethnic groups become more salient, undermining the Black-White contrast. The increasingly complex mapping of the changing social environment may help undermine implicit Blackweapon associations. When, over time, multiple ethnic groups represent more sizable fractions of the local population, the tendency to contrast Black Americans to White Americans may give way to more nuanced distinctions and, as a result, dilute stereotypic assumptions about Black Americans. Following a relatively similar logic, as integration increases over time in a metropolitan area, the Black-White divide may become less accessible and notions of crime, violence, or danger might be less easily associated with Black Americans. This is also likely to be the case because an increase in integration is likely to be accompanied by more frequent, more positive, and less superficial intergroup contact. Individuals immersed in increasingly integrated metropolitan areas are more likely to benefit directly or indirectly from intergroup contact (Christ et al., 2014). Given the more limited role of minority representation (proportion of African Americans) in the cross-sectional examination (Sadler \& Devos, 2020), we did not formulate a hypothesis for this dimension of context diversity, but it was introduced in the model 


\section{CONTEXT DIVERSITY PREDICTS WEAPON BIAS DECLINE}

to partial out any effects it might have. It is possible that, as the relative size of an ethnic minority group increases over time, it becomes more salient in mental representations of the environment, but it is not clear that it would undermine implicit stereotypic associations about criminality. This would only be likely if we assume that minority representation affects the accessibility of counter-stereotypic exemplars.

To test these hypotheses, we utilized a repeated cross-sectional design, i.e., when multiple cross-sections (contexts) have been observed over time with new respondents in each cross-section (Fairbrother, 2014; Ruspini, 1999). More precisely, we relied on an extension of multilevel modeling techniques, introduced by Fairbrother (2014), that separates estimation of cross-sectional and longitudinal effects of a predictor. By clearly distinguishing these two components, we were able to test whether linear trends in context diversity were related to linear changes in implicit Black-weapon associations over time. Adopting this approach represents an important step in the development of a socio-structural perspective on implicit biases as it moves away from static descriptions of relations between contextual characteristics and levels of implicit biases to document dynamic variations over time. In our opinion, a socio-structural perspective should account for both stability and change in implicit biases.

By relying on a multilevel modeling approach, we circumvented the limitations of aggregating data at the context level and were able to control demographic variables. In doing so, we subscribed to a perspective that considers simultaneously the role of context-related and person-related factors (Gawronski \& Bodenhausen, 2017). In the present case, the goal was to demonstrate that contextual factors, both in their stable and transient dimensions, account for implicit associations assessed at the individual level over and above variations attributable to socio-demographic characteristics such as age, gender, ethnicity, level of education, and political 
CONTEXT DIVERSITY PREDICTS WEAPON BIAS DECLINE

orientation. In addition, this analytic approach afforded us the opportunity to examine whether the cross-sectional and longitudinal effects of context diversity were moderated by participant ethnicity. Black respondents typically display weaker implicit Black-weapon associations compared to White respondents (Nosek et al., 2007; Sadler \& Devos, 2020). Unknown from previous work is whether changes in context diversity differentially predict implicit biases among Black vs. White respondents. For Black respondents, this specific implicit bias reflects a negative stereotype associated with the ethnic group they belong to. Without a doubt, the ramifications of social discourse around assumptions of criminality and police brutality are not the same for members of the Black community as they are for other segments of the population. In addition, the increasing diversity of the context they are immersed in may differentially affect individuals as a function of their ethnic identity. In the present study, we explored the moderating role of participant ethnicity to determine whether Black and White respondents were differentially impacted by the changing make-up of the context in which they are immersed.

\section{Method}

\section{Participants}

Participants completed the Weapons IAT on the Project Implicit website (https://implicit.harvard.edu) between January 1, 2009 and December 31, 2018. To be included in the analyses, participants must have obtained a valid IATD score (with no more than $10 \%$ of trial responses faster than 300 milliseconds), provided information on the five sociodemographic variables of interest (i.e., age, sex, race/ethnicity, education attainment, and political orientation), and reported a current zip code located in a Metropolitan Statistical Area (MSA) in the United States. A total of 378,061 participants met the listed criteria. In addition, in order to obtain sound estimates for implicit Black-weapon associations for each year, we only 
CONTEXT DIVERSITY PREDICTS WEAPON BIAS DECLINE

included metropolitan areas that had data for at least 15 participants per year, which reduced the sample by 29,972 (7.9\%) participants. Because the purpose of this study was to examine whether changes in implicit bias over time were related to changes in the ethnic composition in metropolitan areas, participants must have resided in a metropolitan area that had available context diversity data across all 10 years. This further reduced the sample by 2,442 participants (0.7\%). The final sample included a total of 345,647 participants within 185 metropolitan areas (out of 366 possible metropolitan areas). The breakdown of metropolitan areas by U.S. Census regions was as follows: 42 for the West, 52 for the Midwest, 62 for the South, and 29 for the Northeast regions. Besides the steps described in this paragraph, no other exclusion criteria were used.

The average number of participants per metropolitan area was $1,868.36(S D=2,738.36)$. The median number of participants per metropolitan area was 897 (25th percentile $=530.50$, 75th percentile $=1,788.50$, and maximum $=19,316$ ). A majority of the sample consisted of participants who identified as White (68.4\%). The remainder of the sample consisted of $7.4 \%$ Black/African American, 6.2\% Hispanic/Latinx, 5.7\% Asian, and 12.5\% who identified their ethnicity as "Other" or who identified with more than one ethnicity. There was a slight majority of men $(53.9 \%)$ compared to women $(46.1 \%)$. On average, participants were 27.48 years old (SD $=12.22$ ). Of the participants who were at least 25 years of age or older, $64.2 \%$ held at least a bachelor's degree. Lastly, the sample leaned slightly liberal $(M=4.36, S D=1.69$ on a 7-point scale). Considering that the sample consisted mostly of White, relatively young (mid-20s), and relatively educated individuals, it is important to emphasize that it is not representative of the U.S. population.

\section{Sample Size Justification}


CONTEXT DIVERSITY PREDICTS WEAPON BIAS DECLINE

We considered general recommendations about minimum sample sizes for MLM due to the use of existing data. For example, Hox (2010) concluded that 50 groups with 5 cases per group provides sufficient power to test fixed effects, whereas 100 to 200 groups with approximately 10 cases per group is sufficient for random effects and cross-level interactions. According to Snijders (2005), the sample size that matters most is the sample size at which the hypothesized effects are tested. In the present case, Level 2 (Time) is most relevant. Ten years was the maximum number of time points that could be included because American Community Survey (ACS) data necessary to compute the context diversity indicators were not available prior to 2009. Only metropolitan areas that had context diversity information available for all 10 years ${ }^{1}$ were included in the analyses. At Level 3, each metropolitan area had a minimum of 15 observations per year.

To complement the general guidelines, post-hoc sensitivity analyses using simr were performed (Green \& MacLeod, 2016). Currently, sensitivity simulations can be estimated using the observed variable metric (coefficients and their errors), rather than an effect size such as Cohen's $d$ or $r$. The estimated values below can be compared to coefficients reported in Table 1 to evaluate effect magnitudes. To illustrate the sensitivity we had at each level, a predictor of interest was chosen and a 1000-trial simulation was conducted. Sensitivity was $99.50 \%$ to detect a .007 difference in implicit associations of White compared to Black participants at Level 1. Sensitivity was $94.90 \%$ to detect a -.06 interactive effect between time and the longitudinal effect of variety at Level 2. Finally, sensitivity was $97.20 \%$ to detect a -.03 cross-sectional effect of variety at Level 3. Thus, general guidelines and sensitivity analyses suggest that power was sufficient for the study.

\footnotetext{
${ }^{1}$ Relaxing this criterion to include metropolitan areas that had at least 9 out of 10 years resulted in similar findings.
} 
CONTEXT DIVERSITY PREDICTS WEAPON BIAS DECLINE

\section{Procedure}

Participants completed the Weapons IAT, a short questionnaire, and demographic questions of their own accord. The order in which participants completed the measures was counterbalanced. Only measures used to address the aims of the present research are described here. The full dataset with all measures and stimuli can be found at https://osf.io/f4htw/.

Implicit Black-weapon associations. The Weapons IAT measured the strength and direction of the associations between the concepts "weapons" vs. "harmless objects" and two ethnic groups ("Black Americans" vs. "White Americans”). Participants categorized images displayed on their computer screen into one of the four concept categories. Specifically, participants saw images of weapons (e.g., rifle, grenade, sword), harmless objects (e.g., water bottle, camera, wallet), or close-cropped faces of individuals belonging to the target ethnic groups (e.g., Black or White American face). During the critical blocks of trials, participants had to categorize each image into the appropriate category. For example, in one block of trials, the concept "weapons" was paired with "Black Americans" while the concept "harmless objects" was paired with "White Americans" (i.e., stereotype-consistent pairings). In another block of trials, the concept "weapons" was paired with "White Americans" while the concept "harmless objects" was paired with "Black Americans" (i.e., stereotype-inconsistent pairings). The order of the critical blocks was randomized across participants. Participants were asked to sort each stimulus as quickly as possible into its designated category by pressing the appropriate key (i.e., "E" key to indicate that the stimulus belonged to the category on the left side of the computer screen, and "I" key to indicate that the stimulus belonged to the category on the right side of the computer screen). When participants made an error, a red " $\mathrm{X}$ " appeared on the screen. In order to move on to the next trial, participants had to press the correct key. IATD scores reflected the 
CONTEXT DIVERSITY PREDICTS WEAPON BIAS DECLINE

extent to which the concept "weapons" (relative to "harmless objects") was more strongly associated with Black Americans compared to White Americans. Higher IATD scores indicated a stronger implicit Black-weapon association.

Demographic variables. Participants were asked to complete a series of sociodemographic questions. The present research included age, gender, education attainment, political orientation, race/ethnicity, and current zip code. Current zip codes were used to determine residency in U.S. metropolitan areas.

Context diversity measures. Data from the American Community Survey (ACS) for years 2009 to 2018 were utilized to obtain information about context ethnic diversity for U.S. metropolitan areas. MSAs are areas with a population of at least 65,000 people (based on 1-year ACS data) that comprise a metropolitan area and surrounding areas that are socially or economically integrated (U.S. Census Bureau, 2018a). The primary context independent variables were three ethnic diversity indicators - minority representation, variety, and integration — which reflected different aspects of context diversity (for a detail about the computation of these indicators, see Devos \& Sadler, 2019).

Minority representation was operationalized as the proportion of African Americans in a metropolitan area. Higher numbers indicated that the metropolitan area had a larger proportion of African Americans relative to the other five ethnic groups under consideration (Asian, European, Hispanic/Latinx, Other Single-Racial, and Multi-Racial Americans). Due to the measure being highly skewed, a natural log transformation was applied to minority representation in all analyses. Variety was calculated as an entropy score (Iceland, 2004), which reflected the proportional representation of the six ethnic groups per metropolitan area. Variety scores ranged from 0 to 1.792, with higher numbers reflecting more even representation of the six ethnic 
CONTEXT DIVERSITY PREDICTS WEAPON BIAS DECLINE

groups. For example, an area that is made up of 30\% European Americans, 20\% African

Americans, 25\% Asian Americans, 15\% Hispanic/Latinx Americans, 5\% Other Single-Racial

Americans, and 5\% Multi-Racial Americans will have a higher variety score compared to an area that is made up of 50\% European Americans, 20\% African Americans, 10\% Asian Americans, 10\% Hispanic/Latinx Americans, 5\% Other Single-Racial Americans, 5\% Multi-Racial Americans). Minority representation and variety measures were calculated using 1-year ACS data (U.S. Census Bureau, n.d.-a).

In order to calculate the integration measure, variety scores were also computed for census tracts within metropolitan areas. Census tracts are smaller geographical areas with a population of at least 1,200 people that are relatively stable over time (U.S. Census Bureau, 2018b). Because census tract level information is unavailable in the 1-year ACS datasets, the integration measure was calculated using 5-year ACS data (U.S. Census Bureau, n.d.-b). Integration was calculated as an entropy score using the multigroup Theil's $\mathrm{H}$ or multigroup information theory index (Iceland, 2004). Taking into account ethnic diversity information from census tracts, integration scores compared variety scores of census tracts to that of the corresponding metropolitan area (i.e., the distribution of the proportional representation of the six ethnic groups at the census tract level were compared to the corresponding metropolitan area). The integration entropy scores ranged from 0 to 1 , with higher numbers indicating that the diffusion of the ethnic groups at the metropolitan area and census tract levels was similar whereas lower numbers indicated that census tracts were less diverse relative to the corresponding metropolitan area.

To differentiate between cross-sectional and longitudinal effects, two variables were calculated for each of the context diversity measures. The first variable captured the cross- 
CONTEXT DIVERSITY PREDICTS WEAPON BIAS DECLINE

sectional component of the context diversity measures and was computed by calculating each metropolitan area's average level of ethnic diversity over the span of 2009 to 2018 (e.g., $\underline{M R}$ ). Thus, each metropolitan area had its own average level of minority representation, variety, and integration. The cross-sectional component allowed us to test whether a metropolitan area's average level of context diversity was related to implicit Black-weapon associations, collapsing over the years. The second variable for each of the context diversity measures captured the longitudinal component, which represented the change in yearly ethnic diversity (e.g., MRLin ${ }_{\mathrm{tj}}$ ). Simply stated, these time-varying longitudinal context diversity measures were created by calculating each metropolitan area's average level of ethnic diversity and subtracting that average level from the yearly estimates (e.g., $\mathrm{MR}_{\mathrm{tj}}-\underline{M R_{\mathrm{j}}}$ ). Thus, these deviations represented the extent to which context diversity increased or decreased as years passed. Importantly, through the inclusion of the cross-sectional context diversity variables, average levels of context diversity were controlled, which ensured that the longitudinal context diversity variables were capturing only the covariation between changes in ethnic diversity over time and changes in implicit Black-weapon associations over time.

\section{Results}

IATD scores were computed using the algorithm recommended by Greenwald, Nosek, et al. (2003). Higher IATD scores indicated a stronger association between the concept "weapons" (relative to "harmless objects") and Black Americans (compared to White Americans). At the individual level, Black-weapon associations ranged from -1.858 to $1.968(M=0.33, S D=0.39)$.

\section{Descriptive Statistics and Correlations among Context Diversity Measures}

On average, metropolitan areas had low representation of African Americans $(M=0.11$, $S D=0.10)$, were moderately multiethnic $(M=0.89, S D=0.24)$ but were well-integrated $(M=$ 
CONTEXT DIVERSITY PREDICTS WEAPON BIAS DECLINE

$0.82, S D=0.08)$. By examining the bivariate correlations among the three context diversity indicators, there was a relatively strong, positive correlation between minority representation and variety $\left(r_{183}=.51, p<.001\right)$, indicating that metropolitan areas with larger proportions of African Americans were also more multiethnic (i.e., were characterized by greater representation of all other ethnic groups). There was a relatively strong, negative correlation between minority representation and integration $\left(r_{183}=-.53, p<.001\right)$, indicating that metropolitan areas with larger proportions of African Americans were relatively segregated. Lastly, there was a weak, negative correlation between variety and integration $\left(r_{183}=-.21, p=.004\right)$, indicating that more multiethnic metropolitan areas tended to be relatively segregated.

To ensure that we would be able to observe a change in context diversity over the 10year period, we compared the context diversity levels from 2009 to 2018. In 2009, metropolitan areas had low representation of African Americans $(M=0.10, S D=0.10)$, were moderately multiethnic $(M=0.83, S D=0.25)$ and relatively well-integrated $(M=0.85, S D=0.08)$. In 2018, although metropolitan areas continued to have low representation of African Americans $(M=$ $0.11, S D=0.10)$, they were much more multiethnic $(M=0.93, S D=0.23)$ despite being a bit less integrated $(M=0.83, S D=0.08)$. Based on these descriptive statistics, it was notable that only variety experienced a fair amount of change among the context diversity indicators, whereas minority representation and integration remained relatively stable.

\section{Testing the Effects of Time and Context Diversity on Implicit Black-Weapon Associations}

We analyzed the repeated cross-sectional data by applying a three-level multilevel model. Multilevel modeling allowed us to take into account the naturally nested structure of the data (e.g., individuals are part of a shared environment, in this case, their geographical area), ask questions about longitudinal relationships, and investigate context-level characteristics (e.g., 
CONTEXT DIVERSITY PREDICTS WEAPON BIAS DECLINE

ethnic diversity) while controlling for participant socio-demographics (Fairbrother, 2014; Luke, 2004). Specifically, Level 1 included participant demographic variables, Level 2 included timevarying predictors (e.g., longitudinal context diversity indicators), while Level 3 included the cross-sectional context diversity indicators. Individual-level IATD scores served as the dependent variable, participant served as the Level 1 unit, year served as the Level 2 unit, and metropolitan area served as the Level 3 unit. Level 1 and Level 2 fixed effects were group meancentered and Level 3 fixed effects were grand-mean centered (Enders \& Tofighi, 2007).

Currently, there is no consensus on the calculation of effect sizes within multilevel models (Peugh, 2010; Rights \& Sterba, 2019) and the literature is even scarcer when it comes to threelevel models. Despite the lack of agreement on the calculation of effect sizes, it is important to report effect sizes due to their independence from sample size (Schäfer \& Schwarz, 2019), which is especially necessary given the large sample in the present research. For this reason, the interpretation of the findings relied on both significance levels and effect sizes. Following the recommendation by Benjamin et al. (2018), we used an alpha level of .005 to determine significance. Pseudo $R^{2}$ effect sizes were calculated according to Snijders and Bosker (2012), which are standardized effect sizes that can be compared across studies with similar population samples (Lorah, 2018).

We also included a control variable to represent the occurrence of a procedural change to the Weapons IAT. ${ }^{2}$ Because the goal of this study was to examine the longitudinal influences of context diversity, the inclusion of this control variable ensured that any observed relationships

\footnotetext{
2 On August 29, 2016, Block 5 (practice reverse single categorization) was reduced from 40 to 28 trials to attenuate the effect of block order. This change did not affect the number of trials used to compute the IATD but may nonetheless affect the magnitude of the effect.
} 
CONTEXT DIVERSITY PREDICTS WEAPON BIAS DECLINE

between Black-weapon associations, time, and context diversity was not a result of the change in the task. ${ }^{3}$

As reflected in the intercept, evidence of an implicit stereotype was apparent (see Table 1 - Model 1). Specifically, weapons (relative to harmless objects) were more strongly associated with Black Americans than with White Americans. With the exception of education attainment, all participant socio-demographic variables were significant, though the effect sizes were quite small. Black-weapon associations were stronger among older than younger participants $\left(R^{2}=\right.$ $.001)$, among men than women $\left(R^{2}=.0009\right)$, among conservative than liberal participants $\left(R^{2}=\right.$ $.002)$, and among White than Black participants $\left(R^{2}=.011\right)$. Additionally, implicit Blackweapon associations were stronger before the task change compared to after the task change, $R^{2}$ $=.065$. The strength of this effect demonstrates the importance of controlling for task changes, particularly when conducting longitudinal analyses.

In support of Hypothesis 1, there was a decrease in implicit Black-weapon associations over time, $R^{2}=.095$, that was independent of the effects of context diversity (as well as independent of the task change). Moreover, in line with Hypothesis 2, we observed an interaction between time and longitudinal variety, $R^{2}=.019$, demonstrating that as metropolitan areas became more multiethnic over time, Black-weapon associations became weaker over the corresponding time period (see Figure 1). To decompose the interaction, we tested the simple effects, calculated at $1 S D$ above and below the mean. In regard to longitudinal variety, values at +/- $1 S D$ represent faster vs. slower rates of change in variety over the 10-year period. In regard to time, values at $+/-1 S D$ represent specific time points, i.e., mid-2010 and mid-2016. Specifically, decreases in implicit Black-weapon associations over time were greater when

\footnotetext{
${ }^{3}$ In the present study, we could not examine interactions among context diversity indicators at Level 2 due to the limited degrees of freedom.
} 
CONTEXT DIVERSITY PREDICTS WEAPON BIAS DECLINE

metropolitan areas experienced greater increases in variety, $B=-0.015, t=-15.482, p<.001, R^{2}$ $=.091$, compared to when metropolitan areas experienced smaller increases in variety, $B=-$ $0.010, t=-12.033, p<.001, R^{2}=.046$. From the other perspective, earlier in time (i.e., middle of 2010) implicit Black-weapon associations were stronger in metropolitan areas that experienced greater changes in variety over the decade than in metropolitan areas that saw more limited fluctuations in variety, $B=0.343, t=5.065, p<.001, R^{2}=.013$, whereas later in time (i.e., middle of 2016) this difference was no longer reliable, $B=-0.114, t=-1.515, p=.130, R^{2}=$ .002. In contrast to Hypothesis 3, there was no evidence of a similar relationship between time and longitudinal integration. Although we did not have an a priori hypothesis regarding the relationship between time and longitudinal minority representation, it is notable that this effect did not account for changes in Black-weapon associations over time.

In regard to the cross-sectional relationship between context diversity and implicit Blackweapon associations, variety was significantly related to Black-weapon associations, $R^{2}=.036$, such that more multiethnic metropolitan areas were characterized by weaker implicit Blackweapon associations, which is consistent with past research (Sadler \& Devos, 2020). Though the cross-sectional effect of integration was marginal $\left(R^{2}=.008\right)$, the effect also remained consistent with previous findings (Sadler \& Devos, 2020).

Conclusively, the results indicated that the distinction between longitudinal and crosssectional effects of contextual diversity was pertinent. The effect of variety was observed both cross-sectionally and longitudinally (in conjunction with time), but the effect of integration was not. Importantly, and most germane to the aims of the present research, the results provided initial evidence that changes in implicit Black-weapon associations over time depended on the 
CONTEXT DIVERSITY PREDICTS WEAPON BIAS DECLINE

pattern of change in context diversity, specifically in the degree of proportional representation of ethnic groups within metropolitan areas. ${ }^{4}$

\section{Testing for Moderation by Participant Ethnicity}

To examine whether the relationship between cross-sectional and longitudinal context diversity and implicit Black-weapon associations differed as a function of participant ethnicity, we set the slope for participant ethnicity as random at both Level 2 and Level 3 and added crosslevel interactions between participant ethnicity (contrast between Black and White participants) and all Level 2 and Level 3 covariates (see Table 1 - Model 2). Only the cross-level interaction between the White vs. Black contrast and time was significant $\left(R^{2}=.010\right)$. To decompose this interaction, we ran the model separately for White vs. Black participants, ${ }^{5}$ which indicated that Black-weapon associations were weakening at a faster rate for Black participants, $B=-0.021, t=$ $-8.167, p<.001, R^{2}=.272$, compared to White participants, $B=-0.010, t=-12.054, p<.001, R^{2}$ $=.062$. Moreover, the pattern of results from the previous model held. Thus, although participant ethnicity did explain additional variation in implicit Black-weapon associations, this did not qualify the main findings.

\section{Discussion}

The present research tested whether the association between weapons and Black Americans decreased over time and whether temporal changes in this stereotype were related to changes in context diversity over the same time period. As hypothesized, the analyses documented a weakening of Black-weapon associations over time (Hypothesis 1), as well as a

\footnotetext{
${ }^{4}$ We also ran an analysis with more liberal inclusion criteria. Specifically, we included metropolitan areas that had at least 30 participants over the 10-year period (instead of at least 15 participants for each year). This resulted in a total of 372,228 participants within 331 metropolitan areas. The results were comparable to those reported here. ${ }^{5}$ Although the model converged for Black participants, we received a warning that the final Hessian matrix was not positive definite, meaning that the obtained estimates were not stable. Thus, we report these findings with caution.
} 
CONTEXT DIVERSITY PREDICTS WEAPON BIAS DECLINE

longitudinal relationship between variety and implicit Black-weapon associations (Hypothesis 2).

Specifically, as metropolitan areas become more multiethnic over time, implicit Black-weapon

associations become weaker over time. In contrast to what we predicted (Hypothesis 3),

decreases in implicit Black-weapon associations did not depend on longitudinal changes in integration.

Overall, these results suggest that the distribution of multiple ethnic groups in a context plays a role in the level of implicit bias within a metropolitan area (compared to the growth of a single minority group or the discrepancy in the ethnic distribution when comparing neighborhoods to the broader context). In and of itself, the specificity of relation between change in context variety and implicit Black-weapon associations is evidence for the usefulness of simultaneously examining distinct facets of context diversity. Multiple mechanisms might account for this relation. Taking as a starting point the notion that implicit biases reflect the knowledge that is accessible in a given context at a specific point in time, individuals are likely to be exposed to information and have experiences that differ depending on how multiethnic their environment is. Everyday interactions with others, exposure to ideas, practices, symbols, or products may change as the population reflects a more even representation of various ethnic groups. We posit that one of potentially many implications of increasing multiethnic metropolitan areas is that distinctions among multiple ethnic groups become more salient, undermining the Black-White contrast. The increasingly complex mapping of the social environment may undermine implicit Black-weapon associations. As more nuanced distinctions between fractions of the local population come to the forefront, stereotypic assumptions about criminality, danger, or violence and Black Americans may weaken. We had anticipated that integration would play on similar processes with potentially the added influence of direct and 
CONTEXT DIVERSITY PREDICTS WEAPON BIAS DECLINE

indirect intergroup contact associated with increasingly integrated metropolitan areas. Although we replicated the cross-sectional effect of integration (Sadler \& Devos, 2020), we did not find support for a longitudinal effect of this facet of context diversity. This could result from the relatively limited changes in integration in comparison to changes in variety (see also Limitations section). Once again, minority representation was not a source of reliable effects. Given that this was the case at both the cross-sectional and longitudinal levels, we conclude that although Black Americans as an ethnic group may become more salient when they represent an increasingly large segment of the metropolitan population, this does not translate into a weakening of implicit stereotypes.

The use of a repeated cross-sectional design allows for the ability to distinguish between longitudinal and cross-sectional effects of context diversity within the same model (Fairbrother, 2014). This analytic framework is suitable to document changes over time in implicit Black-weapon associations without compromising the assumptions of linearity and independence (Rafferty et al., 2015). Using this novel technique, this study is the first to provide evidence of a longitudinal relationship between context diversity and implicit bias while simultaneously controlling for the cross-sectional relationship. The implicit stereotypic association between Blacks and weapons is mitigated progressively as contexts become more multiethnic. In regard to cross-sectional relationships, metropolitan areas in which ethnic groups are more evenly distributed (i.e., higher variety) and in which the distributions of the ethnic groups at the census tract and metropolitan area levels are more similar (i.e., higher integration) have lower levels of implicit Black-weapon associations. Both effects are consistent with past research (Sadler \& Devos, 2020). 
CONTEXT DIVERSITY PREDICTS WEAPON BIAS DECLINE

The literature on the role of context diversity in intergroup relations points to contrasting effects: perceived or actual increases in diversity might be associated with a sense of threat that intensifies intergroup biases (Craig \& Richeson, 2014; Craig et al., 2018), but could also provide opportunities for intergroup contact that, under optimal conditions, typically reduce intergroup biases (Christ et al., 2014; Hewstone, 2015; Schmid et al., 2013). In the realm of implicit biases, this tension also emerges in that context diversity can be associated either with stronger (Rae et al., 2015) or weaker (Devos \& Sadler, 2019; Sadler \& Devos, 2020) intergroup biases. Scarce attention has been paid to the impact of actual change in context diversity (cf. Devos et al., 2019; Ramos et al., 2019). The present study contributes to fill this gap. Although the literature is too scant to develop a solid account of the conditions under which increasing context diversity intensifies or mitigates intergroup biases, one avenue to integrate available findings is to draw a distinction between the perceived and actual diversity of contexts and to compare reactions documented using self-report measures vs. implicit measures. Threat responses and stronger intergroup biases tend to be associated with subjective perceptions of change in context diversity and to transpire from self-reported attitudes or opinions. Evidence for weaker intergroup biases tend to emerge more often when the actual diversity of contexts is assessed and on implicit measures of attitudes or stereotypes.

More broadly, the present work supports recent arguments to examine socio-structural factors as potential determinants of both stability and fluctuation in implicit bias over time (Daumeyer et al., 2017; Payne \& Vuletich, 2018; Payne et al., 2017). There have only been a handful of studies examining the role of socio-structural factors, such as contextual ethnic diversity (Devos \& Sadler, 2019; Devos et al., 2019; Rae et al., 2015; Sadler \& Devos, 2020), legalization of same-sex marriage (Ofosu et al., 2019), and proportion of slaves in 1860 (Payne 


\section{CONTEXT DIVERSITY PREDICTS WEAPON BIAS DECLINE}

et al., 2019). Independent from the changes in context diversity, the strength of the decrease in implicit Black-weapon associations over time suggests that other factors may mitigate this bias. Media coverage and social media platforms have contributed to a greater amount of public attention related to the disproportionate police violence against Black Americans, potentially resulting in the weakening of implicit Black-weapon associations (Charlesworth \& Banaji, 2019). Other possible factors influencing the strength of implicit Black-weapon associations might be the BLM movement (Sawyer \& Gampa, 2018), law enforcement policies, and police force diversity (McElvain \& Kposowa, 2008; Rosenthal, 2016). Although the exact mechanisms underlying effects reported here are not fully understood, the present findings are consistent with prior research documenting that implicit racial biases have declined over time (Charlesworth \& Banaji, 2019; Sawyer \& Gampa, 2018). The novelty here is to provide evidence for temporal fluctuations in the domain of stereotypes (Black-weapon associations). Thus, change in implicit racial biases are not restricted to attitudinal or evaluative associations. Although the stability of implicit racial biases is also worth noting (Payne et al., 2019), evidence for lack of change could stem from suboptimal data analytic approaches (Schmidt \& Axt, 2016; Schmidt \& Nosek, 2010).

\section{Limitations}

Several limitations of the present study are worth acknowledging. First, despite the large sample size, participants self-selected into completing the Weapons IAT. Thus, the sample is a convenience sample rather than a random or representative sample. Although it was relatively heterogeneous and allowed us to control for the effects of important socio-demographic variables, the fact that it was mostly composed of relatively young, educated, liberal, and White respondents limits our ability to generalize the findings. This being said, several important points pertaining to the non-representativeness of the sample are worth mentioning. To begin, the issue 
CONTEXT DIVERSITY PREDICTS WEAPON BIAS DECLINE

of representativeness is not central to the aims of the present research in the sense that we are not making inferences about population estimates. Even when respondents are not representative of a given population, it might be possible to document meaningful contextual and temporal fluctuations. The outcome of the analyses support this assumption. In addition, it is worth noting that socio-demographic variables were introduced in all analyses and yielded relatively tenuous effects. At a minimum, context-level factors are sources of detectable effects over and above individual-level characteristics. We did not systematically examine cross-level interactions, but when we did for what is likely to be the most relevant social identity (i.e., respondents' ethnicity) the outcome of the analyses indicated that this factor moderated the magnitude, but not the direction of the effects (i.e., overall weaker Black-weapon associations and stronger decline over time among Black rather than White respondents). Finally, although the sample included respondents from distinct regions of the U.S., the coverage was limited to metropolitan areas. Thus, the findings cannot be generalized to less densely populated and more rural areas.

A second important limitation is that, despite modeling a longitudinal trend, the design does not allow us to infer causality. We are unable to determine whether changes in context diversity cause changes in implicit Black-weapon associations, whether changes in implicit Black-weapon associations lead to changes in context diversity, or whether an unobserved variable yields changes in both implicit Black-weapon associations and context diversity. The assumption behind our theorizing is that socio-structural changes produce changes in implicit biases, but alternative causal pathways are worth considering. For example, people may selectively move to certain areas based on the perceived or actual level of implicit bias, potentially resulting in greater ethnic variety. 
CONTEXT DIVERSITY PREDICTS WEAPON BIAS DECLINE

Lastly, the lack of evidence for the role of integration might partially be attributed to a limitation of the measure used. Ideally, yearly integration would be calculated from yearly tractlevel information. However, racial-ethnic information at the tract level is only available as a 5year rolling average (U.S. Census Bureau, 2019). This is suboptimal because it could underestimate the degree of change from one year to the next (e.g., average score computed from 2009-2014 compared to average score computed from 2010-2015). The usage of 5-year data might explain why we do not observe a longitudinal effect of integration in the present study.

\section{Conclusion}

The present research adds to the burgeoning literature examining socio-structural factors as a basis for understanding variation in implicit biases. The findings show that as metropolitan areas become more multiethnic over time, implicit associations between weapons and Black Americans weaken. The extent of change in implicit stereotypes over time is partially explained by context diversity, but additional factors are likely contributing to this weakening trend. To our knowledge, this study is the first to demonstrate that socio-structural changes within an area over time correspond to changes in implicit biases in this area. Socio-structural accounts of implicit biases have shifted the focus from studying mental associations residing within individuals to stressing their societal and systemic foundations. Empirically, one way of doing so is to document that socio-structural characteristics of contexts, such as context diversity, predict the magnitude of implicit biases. Rather than prioritizing one level of analysis over another, we think that the value and potential implications of this framework lies in acknowledging the interconnections between individual-level processes and socio-structural dynamics. We cannot understand implicit biases displayed by individuals without considering the contexts in which these individuals are immersed, the regularities in their experiences and in the knowledge that is 
CONTEXT DIVERSITY PREDICTS WEAPON BIAS DECLINE

accessible to them on a daily basis. Implicit biases are the thumbprint of systemic relations between groups at the societal level, but also at the more local level (metropolitan areas, cities, or communities). Because socio-structural realities tend to be stable over time, this perspective often points to the ineluctability of implicit biases, but the temporal variations over a relatively short period of time (one decade) documented in the present research, and in particular the ability to tie these variations to the changing nature of the contexts (increasing ethnic variety), stresses the importance of understanding the systems (e.g., institutions, policies, medias) that perpetuate or change the contexts in which individuals are reared. In the current socio-political context, this perspective may have important practical implications. Formulating specific solutions or recommendations would be ill-advised, but taking implicit biases as a window into systemic forces might be a promising prism to address deeply entrenched racial injustices and forms of oppressions. 
CONTEXT DIVERSITY PREDICTS WEAPON BIAS DECLINE

\section{References}

Benjamin, D. J., Berger, J. O., Johannesson, M., Nosek, B. A., Wagenmakers, E. J., Berk, R., Bollen, K. A., Brembs, B., Brown, L., Camerer, C., Cesarini, D., Chambers, C. D., Clyde, M., Cook, T. D., De Boeck, P., Dienes, Z., Dreber, A., Easwaran, K., Efferson, . . . Johnson, V. E. (2018). Redefine statistical significance. Nature Human Behaviour, 2(1), 6-10. https://doi.org/10.1038/s41562-017-0189-z

Charlesworth, T. E. S., \& Banaji, M. R. (2019). Patterns of implicit and explicit attitudes: I. Long-term change and stability from 2007 to 2016. Psychological Science, 30(2), 174192. https://doi.org/10.1177/0956797618813087

Christ, O., Schmid, K., Lolliot, S., Swart, H., Stolle, D., Tausch, N., Al Ramiah, A., Wagner, U., Vertovec, S., \& Hewstone, M. (2014). Contextual effect of positive intergroup contact on outgroup prejudice. Proceedings of the National Academy of Sciences, 111(11), 39964000. https://doi.org/10.1073/pnas.1320901111

Cooley, E., Hester, N., Cipolli, W., Rivera, L. I., Abrams, K., Pagan, J., Sommers, S. R., \& Payne, B. K. (2019). Racial biases in officers' decisions to frisk are amplified for Black people stopped among groups leading to similar biases in searches, arrests, and use of force. Social Psychological and Personality Science, 11(6), 761-769. https://doi.org/10.1177/1948550619876638

Correll, J., Hudson, S. M., Guillermo, S., \& Ma, D. S. (2014). The police officer's dilemma: A decade of research on racial bias in the decision to shoot. Social and Personality Psychology Compass, 8(5), 201-213. https://doi.org/10.1111/spc3.12099 
CONTEXT DIVERSITY PREDICTS WEAPON BIAS DECLINE

Correll, J., Park, B., Judd, C. M., \& Wittenbrink, B. (2002). The police officer's dilemma: Using ethnicity to disambiguate potentially threatening individuals. Journal of Personality and Social Psychology, 83(6), 1314-1329. https://doi.org/10.1037/0022-3514.83.6.1314

Correll, J., Wittenbrink, B., Park, B., Judd, C. M., \& Goyle, A. (2011). Dangerous enough: Moderating racial bias with contextual threat cues. Journal of Experimental Social Psychology, 47(1), 184-189. https://doi.org/10.1016/j.jesp.2010.08.017

Craig, M. A., \& Richeson, J. A. (2014). More diverse yet less tolerant? How the increasingly diverse racial landscape affects White Americans' racial attitudes. Personality and Social Psychology Bulletin, 40(6), 750-761. https://doi.org/10.1177/0146167214524993

Craig, M. A., Rucker, J. M., \& Richeson, J. A. (2018). The pitfalls and promise of increasing racial diversity: Threat, contact, and race relations in the 21 st century. Current Directions in Psychological Science, 27(3), 188-193. https://doi.org/10.1177/0963721417727860

Daumeyer, N. M., Rucker, J. M., \& Richeson, J. A. (2017). Thinking structurally about implicit bias: Some peril, lots of promise. Psychological Inquiry, 28(4), 258-261. https://doi.org/10.1080/1047840X.2017.1373556

Devos, T., \& Sadler, M. (2019). Context diversity predicts the extent to which the American identity is implicitly associated with Asian Americans and European Americans. Asian American Journal of Psychology, 10(2), 182-193. https://doi.org/10.1037/aap0000149

Devos, T., Sadler, M., Perry, D., \& Yogeeswaran, K. (2019). Temporal fluctuations in context ethnic diversity over three decades predict implicit national inclusion of Asian Americans. Group Processes \& Intergroup Relations. https://doi.org/10.1177/1368430219887440 
CONTEXT DIVERSITY PREDICTS WEAPON BIAS DECLINE

Eberhardt, J. L., Goff, P. A., Purdie, V. J., \& Davies, P. G. (2004). Seeing Black: Race, crime, and visual processing. Journal of Personality and Social Psychology, 87(6), 876-893. https://doi.org/10.1037/0022-3514.87.6.876

Enders, C. K., \& Tofighi, D. (2007). Centering predictor variables in cross-sectional multilevel models: A new look at an old issue. Psychological Methods, 12(2), 121-138. https://doi.org/10.1037/1082-989X.12.2.121

Fairbrother, M. (2014). Two multilevel modeling techniques for analyzing comparative longitudinal survey datasets. Political Science Research and Methods, 2(1), 119-140. https://doi.org/10.1017/psrm.2013.24

Gawronski, B., \& Bodenhausen, G. V. (2017). Beyond persons and situations: An interactionist approach to understanding implicit bias. Psychological Inquiry, 28(4), 268-272. https://doi.org/10.1080/1047840X.2017.1373546

Green, P., \& MacLeod, C. J. (2016). SIMR: An R package for power analysis of generalized linear mixed models by simulation. Methods in Ecology and Evolution, 7(4), 493-498. https://doi.org/10.1111/2041-210X.12504

Greenwald, A. G., Nosek, B. A., \& Banaji, M. R. (2003). Understanding and using the Implicit Association Test: I. An improved scoring algorithm. Journal of Personality and Social Psychology, 85(2), 197-216. https://doi.org/10.1037/0022-3514.85.2.197

Greenwald, A. G., Oakes, M. A., \& Hoffman, H. G. (2003). Targets of discrimination: Effects of race on responses to weapons holders. Journal of Experimental Social Psychology, 39(4), 399-405. https://doi.org/10.1016/S0022-1031(03)00020-9 
CONTEXT DIVERSITY PREDICTS WEAPON BIAS DECLINE

Hehman, E., Flake, J. K., \& Calanchini, J. (2018). Disproportionate use of lethal force in policing is associated with regional racial biases of residents. Social Psychological and Personality Science, 9(4), 393-401. https://doi.org/10.1177/1948550617711229

Hewstone, M. (2015). Consequences of diversity for social cohesion and prejudice: The missing dimension of intergroup contact. Journal of Social Issues, 71(2), 417-438. https://doi.org/10.1111/josi.12120

Hox, J. (2010). Multilevel analysis: Techniques and applications ( $2^{\text {nd }}$ ed.). New York: Routledge.

Iceland, J. (2004). Beyond Black and White: Metropolitan residential segregation in multi-ethnic America. Social Science Research, 33(2), 248-271. https://doi.org/10.1016/S0049$\underline{089 X(03) 00056-5}$

Inbar, Y., Westgate, E. C., Pizarro, D. A., \& Nosek, B. A. (2016). Can a naturally occurring pathogen threat change social attitudes? Evaluations of gay men and lesbians during the 2014 Ebola epidemic. Social Psychological and Personality Science, 7(5), 420-427. https://doi.org/10.1177/1948550616639651

Jebb, A. T., Tay, L., Wang, W., \& Huang, Q. (2015). Time series analysis for psychological research: Examining and forecasting change. Frontiers in Psychology, 6. https://doi.org/10.3389/fpsyg.2015.00727

Johnson, D. J., \& Chopik, W. J. (2019). Geographic variation in the Black-violence stereotype. Social Psychological and Personality Science, 10(3), 287-294. https://doi.org/10.1177/1948550617753522 
CONTEXT DIVERSITY PREDICTS WEAPON BIAS DECLINE

Koopmans, R., \& Schaeffer, M. (2015). Relational diversity and neighbourhood cohesion.

Unpacking variety, balance and in-group size. Social Science Research, 53, 162-176. https://doi.org/10.1016/j.ssresearch.2015.05.010

Lorah, J. (2018). Effect size measures for multilevel models: Definition, interpretation, and TIMSS example. Large-Scale Assessments in Education, 6.

https://doi.org/10.1186/s40536-018-0061-2

Luke, D. A. (2004). Multilevel modeling. Quantitative applications in the social sciences. Sage. https://doi.org/10.4135/9781412985147

Ma, D. S., \& Correll, J. (2011). Target prototypicality moderates racial bias in the decision to shoot. Journal of Experimental Social Psychology, 47(2), 391-396. https://doi.org/10.1016/j.jesp.2010.11.002

McElvain, J. P., \& Kposowa, A. J. (2008). Police officer characteristics and the likelihood of using deadly force. Criminal Justice and Behavior, 35(4), 505-521. https://doi.org/10.1177/0093854807313995

Mekawi, Y., \& Bresin, K. (2015). Is the evidence from racial bias shooting task studies a smoking gun? Results from a meta-analysis. Journal of Experimental Social Psychology, 61, 120-130. https://doi.org/10.1016/j.jesp.2015.08.002

Murphy, M. C., Kroeper, K. M., \& Ozier, E. M. (2018). Prejudiced places: How contexts shape inequality and how policy can change them. Policy Insights from the Behavioral and Brain Sciences, 5(1), 66-74. https://doi.org/10.1177/2372732217748671

Nosek, B. A., Smyth, F. L., Hansen, J. J., Devos, T., Lindner, N. M., Ranganath, K. A., Smith, C. T., Olson, K. R., Chugh, D., Greenwald, A. G., \& Banaji, M. R. (2007). Pervasiveness 
CONTEXT DIVERSITY PREDICTS WEAPON BIAS DECLINE

and correlates of implicit attitudes and stereotypes. European Review of Social Psychology, 18(1), 36-88. https://doi.org/10.1080/10463280701489053

Ofosu, E. K., Chambers, M. K., Chen, J. M., \& Hehman, E. (2019). Same-sex marriage legalization associated with reduced implicit and explicit antigay bias. Proceedings of the National Academy of Sciences, 116(18), 8846-8851.

https://doi.org/10.1073/pnas.1806000116

Payne, B. K. (2001). Prejudice and perception: The role of automatic and controlled processes in misperceiving a weapon. Journal of Personality and Social Psychology, 81(2), 181-192. https://doi.org/10.1037/0022-3514.81.2.181

Payne, B. K. (2006). Weapon bias: Split-second decisions and unintended stereotyping. Current Directions in Psychological Science, 15(6), 287-291. https://doi.org/10.1111/j.1467$\underline{8721.2006 .00454 . x}$

Payne, B. K., Lambert, A. J., \& Jacoby, L. L. (2002). Best laid plans: Effects of goals on accessibility bias and cognitive control in race-based misperceptions of weapons. Journal of Experimental Social Psychology, 38(4), 384-396. https://doi.org/10.1016/S0022-

\section{$\underline{1031(02) 00006-9}$}

Payne, B. K., \& Vuletich, H. A. (2018). Policy insights from advances in implicit bias research. Policy Insights from the Behavioral and Brain Sciences, 5(1), 49-56. https://doi.org/10.1177/2372732217746190

Payne, B. K., Vuletich, H. A., \& Brown-Iannuzzi, J. L. (2019). Historical roots of implicit bias in slavery. Proceedings of the National Academy of Sciences, 116(24), 11693-11698. https://doi.org/10.1073/pnas.1818816116 
CONTEXT DIVERSITY PREDICTS WEAPON BIAS DECLINE

Payne, B. K., Vuletich, H. A., \& Lundberg, K. B. (2017). The bias of crowds: How implicit bias bridges personal and systemic prejudice. Psychological Inquiry, 28(4), 233-248. https://doi.org/10.1080/1047840X.2017.1335568

Peugh, J. L. (2010). A practical guide to multilevel modeling. Journal of School Psychology, 48(1), 85-112. https://doi.org/10.1016/j.jsp.2009.09.002

Plant, E. A., \& Peruche, B. M. (2005). The consequences of race for police officers' responses to criminal suspects. Psychological Science, 16(3), 180-183. https://doi.org/10.1111/j.09567976.2005.00800.x

Rae, J. R., Newheiser, A.-K., \& Olson, K. R. (2015). Exposure to racial out-groups and implicit race bias in the United States. Social Psychological and Personality Science, 6(5), 535543. https://doi.org/10.1177/1948550614567357

Rafferty, A., Walthery, P., \& King-Hele, S. (2015). Analysing change over time: Repeated cross sectional and longitudinal survey data. https://www.ukdataservice.ac.uk/media/455362/changeovertime.pdf

Ramos, M. R., Bennett, M. R., Massey, D. S., \& Hewstone, M. (2019). Humans adapt to social diversity over time. Proceedings of the National Academy of Sciences, 116(25), 1224412249. https://doi.org/10.1073/pnas.1818884116

Reardon, S. F., \& Firebaugh, G. (2002). Measures of multigroup segregation. Sociological Methodology, 32(1), 33-67. https://doi.org/10.1111/1467-9531.00110

Rights, J. D., \& Sterba, S. K. (2019). Quantifying explained variance in multilevel models: An integrative framework for defining R-squared measures. Psychological Methods, 24(3), 309-338. https://doi.org/10.1037/met0000184 
CONTEXT DIVERSITY PREDICTS WEAPON BIAS DECLINE

Rosenthal, L. (2016). Good and bad ways to address police violence. The Urban Lawyer, 48(4), 675-736. https://ssrn.com/abstract=2864072

Ruspini, E. (1999). Longitudinal research and the analysis of social change. Quality and Quantity, 33, 219-227. https://doi.org/10.1023/A:1004692619235

Sadler, M., \& Devos, T. (2020). Ethnic diversity matters: Putting implicit associations between weapons and ethnicity in context. Group Processes \& Intergroup Relations, 23(2), 285300. https://doi.org/10.1177/1368430218796933

Sawyer, J., \& Gampa, A. (2018). Implicit and explicit racial attitudes changed during Black Lives Matter. Personality and Social Psychology Bulletin, 44(7), 1039-1059. https://doi.org/10.1177/0146167218757454

Schäfer, T., \& Schwarz, M. A. (2019). The meaningfulness of effect sizes in psychological research: Differences between sub-disciplines and the impact of potential biases. Frontiers in Psychology, 10. https://doi.org/10.3389/fpsyg.2019.00813

Schmid, K., Hewstone, M., \& Ramiah, A. A. (2013). Neighborhood diversity and social identity complexity: Implications for intergroup relations. Social Psychological and Personality Science, 4(2), 135-142. https://doi.org/10.1177/1948550612446972

Schmidt, K., \& Axt, J. R. (2016). Implicit and explicit attitudes toward African Americans and Barack Obama did not substantively change during Obama's presidency. Social Cognition, 34(6), 559-588. https://doi.org/10.1521/soco.2016.34.6.559

Schmidt, K., \& Nosek, B. A. (2010). Implicit (and explicit) racial attitudes barely changed during Barack Obama’s presidential campaign and early presidency. Journal of Experimental Social Psychology, 46(2), 308-314.

https://doi.org/10.1016/j.jesp.2009.12.003 
CONTEXT DIVERSITY PREDICTS WEAPON BIAS DECLINE

Snijders, T. A. B. (2005). Power and sample size in multilevel linear models. In B. S. Everitt \& D. C. Howell (Eds.), Encyclopedia of Statistics in Behavioral Science (Vol. 3, pp. 15601573). Wiley. https://doi.org/10.1002/0470013192.bsa492

Snijders, T. A. B., \& Bosker, R. J. (2012). Multilevel analysis: An introduction to basic and advanced multilevel modeling (2nd ed.). Sage.

Sommers, S. R., \& Marotta, S. A. (2014). Racial disparities in legal outcomes: On policing, charging decisions, and criminal trial proceedings. Policy Insights from the Behavioral and Brain Sciences, 1(1), 103-111. https://doi.org/10.1177/2372732214548431

Spencer, K. B., Charbonneau, A. K., \& Glaser, J. (2016). Implicit bias and policing. Social and Personality Psychology Compass, 10(1), 50-63. https://doi.org/10.1111/spc3.12210

U.S. Census Bureau. (2018a). American Community Survey (ACS): When to use 1-year, 3-year, or 5-year estimates. https://www.census.gov/programssurveys/acs/guidance/estimates.html

U.S. Census Bureau. (2018b). Geography Program: Glossary - Census Tract. https://www.census.gov/programs-surveys/geography/about/glossary.html

U.S. Census Bureau. (2019). Comparing ACS Data. https://www.census.gov/programssurveys/acs/guidance/comparing-acs-data.html

U.S. Census Bureau. (n.d.-a). 1-year ACS demographic and housing estimates [Dataset]. https://data.census.gov/cedsci/table?q=DP05\&hidePreview=false\&tid=ACSDP1Y2018.D $\underline{\mathrm{P} 05}$

U.S. Census Bureau. (n.d.-b). 5-year ACS demographic and housing estimates [Dataset]. https://data.census.gov/cedsci/table?q=DP05\&hidePreview=false\&tid=ACSDP5Y2018.D $\underline{\mathrm{P} 05}$ 
CONTEXT DIVERSITY PREDICTS WEAPON BIAS DECLINE

Westgate, E. C., Riskind, R. G., \& Nosek, B. A. (2015). Implicit preferences for straight people over lesbian women and gay men weakened from 2006 to 2013. Collabra, 1(1), 1-10. https://doi.org/10.1525/collabra.18 
CONTEXT DIVERSITY PREDICTS WEAPON BIAS DECLINE

Table 1

Implicit Black-Weapon Associations as a Function of Time, Context Diversity, and Participant Ethnicity

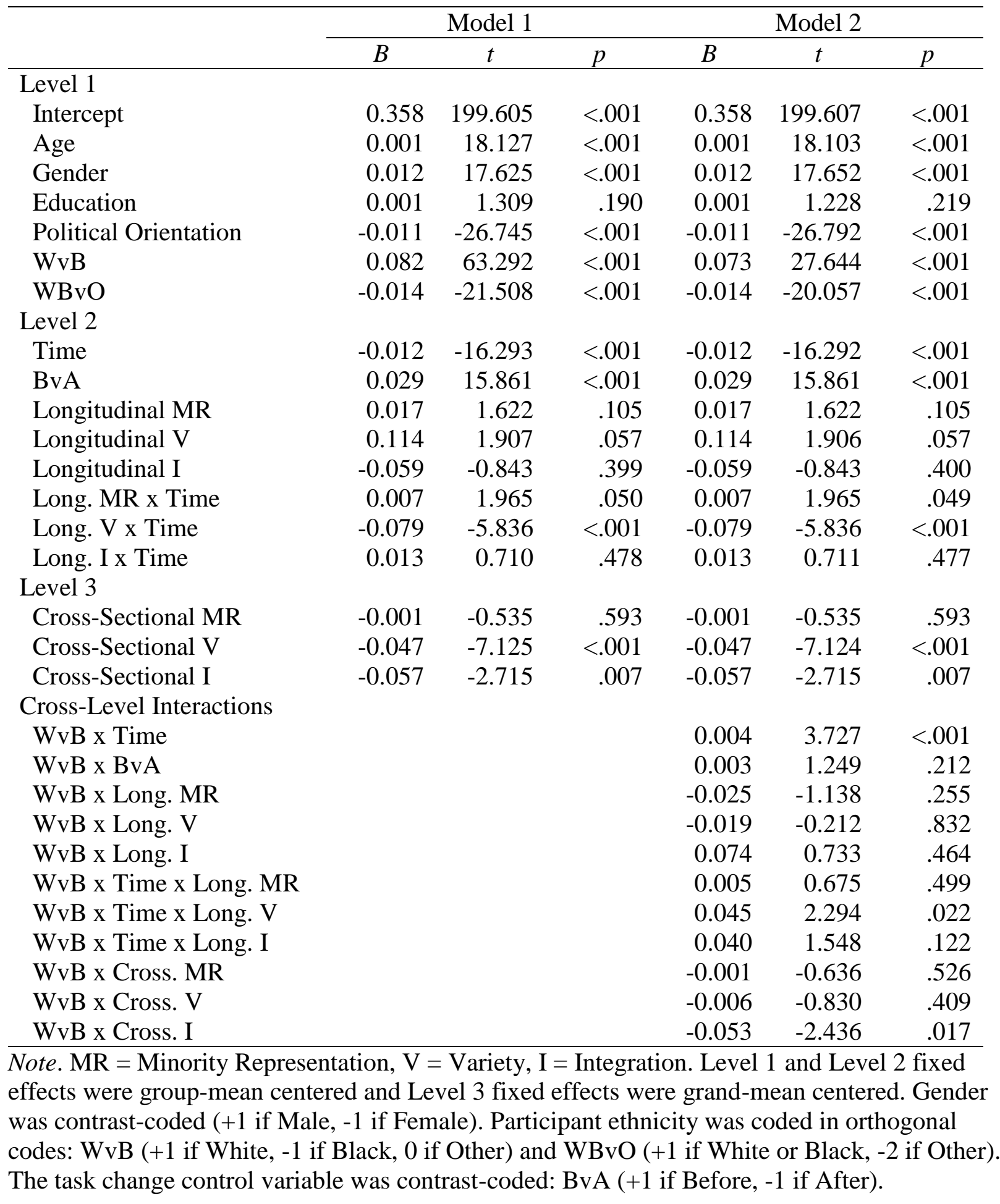


CONTEXT DIVERSITY PREDICTS WEAPON BIAS DECLINE

\section{Figure 1}

Implicit Black-weapon associations as a function of longitudinal variety (i.e., small vs. large increase) and time (i.e., year).

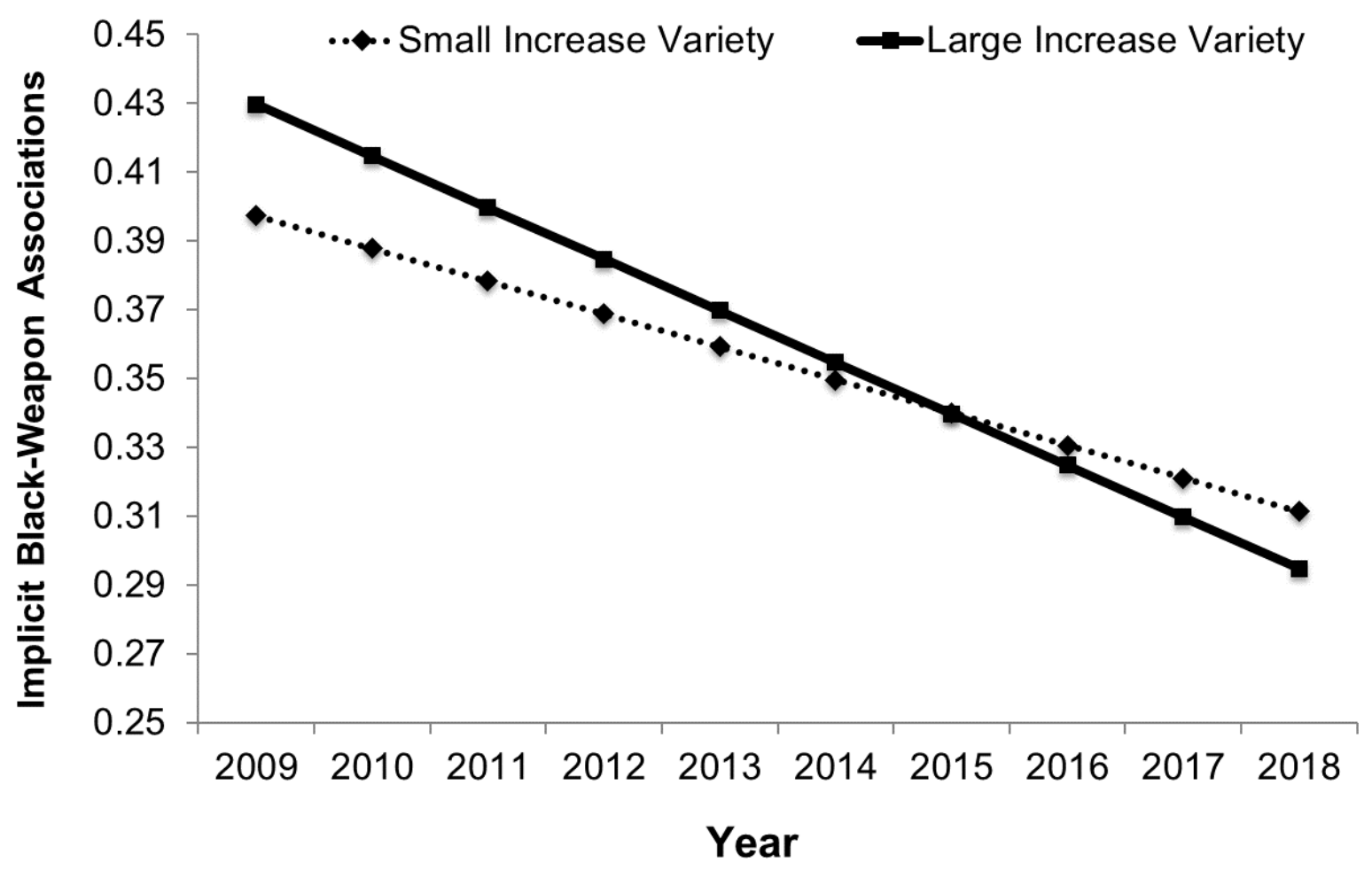

